\title{
Preliminary Clinical Trial of Gadodiamide Injection: A New Nonionic Gadolinium Contrast Agent for MR Imaging ${ }^{1}$
}

\author{
Gabriela D. Kaplan, MD² • Alex M. Aisen, MD • S. Rao Aravapalli, MD ${ }^{3}$
}

The safety and efficacy of a newly developed intravenous formulation of the nonionic contrast agent gadolinium diethylenetriaminepentaacetic acid-bis(methylamide), formulated as gadodiamide injection, was investigated. In 30 patients who underwent spin-echo magnetic resonance (MR) imaging before and after contrast agent enhancement, the enhanced images had characteristics judged similar to those of images enhanced by means of available gadolinium compounds. In 15 patients, contrast agent administration was of major diagnostic help, either revealing lesions not apparent without enhancement or providing important lesion characterization. In 12 patients, the lack of abnormal enhancement patterns was important in excluding the presence of disease. In three patients, the contrast agent did not provide information additional to that obtained with the unenhanced T1- and T2-weighted images. No clinically significant changes were observed in vital signs, neurologic status, or laboratory results. The authors conclude that, in this limited series, gadodiamide injection proved to be a safe and useful MR imaging contrast agent for evaluation of the central nervous system and surrounding structures.

Index terms: Brain neoplasms, MR studies, $10.1214 \cdot$ Brain neoplasms. diagnosis, $10.36,10.38,10.1214 \cdot$ Brain neoplasms, secondary, 10.38 - Contrast enhancement • Gadolinium - Head, MR studies, 10.1214 - Head and neck, neoplasms, 10.3 - Spine, MR studies, 30.1214 • Spine, primary neoplasms, 30.3 - Splne, secondary neoplasms, 30.3

JMRI 1991; 1:57-62

Abbreviations: DOTA = tetraazacyclododecanetetraacetic acid, DTPA-BMA = diethylenetriaminepentaacetic acid-bis (methylamide).

1 From the Department of Radiology, University of Michigan. 1500 E Medical Center Dr, Ann Arbor, MJ 48109-0030. From the 1990 SMRI annual meeting. Received August 20, 1990: revision requested September 27; revision received and accepted October 18. Supported by funding from Salutar, Inc. Address reprint requests to A.M.A.

2 Current address: Department of Radiology, Akron General Medical Center, Akron, Ohio.

${ }^{3}$ Current address: Department of Radiology. SUNY Health Sciences Center, Syracuse, NY

(c) SMRI, 1991
THIS MULTICENTER STUDY EVALUATED the imaging efficacy and clinical safety of a new nonionic gadolinium chelate contrast agent for magnetic resonance (MR) imaging, gadolinium diethylenetriaminepentaacetic acid-bis(methylamide) (abbreviated Gd-DTPA-BMA). Formulated as gadodiamide injection, it was developed by Salutar (Sunnyvale, Calif). Animal studies suggested that because of its nonionic nature and strong chelation (median lethal dose in mice, $34.4 \mathrm{mmol} / \mathrm{kg}$ ), it will likely prove to be a safe MR contrast agent (Salutar, data on file, 1989); this was also supported by the phase I trial of the agent (1).

\section{- MATERIALS AND METHODS}

Patients were selected from those referred for MR imaging with a known or suspected diagnosis of a space-occupying lesion in or adjacent to the central nervous system. Patients were not required to abstain from food or medications before the examination. Informed consent was obtained in each case, and the protocol conformed to U.S. Food and Drug Administration guidelines and was approved by the University of Michigan Institutional Review Board.

A brief neurologic examination was performed, and medical history, vital signs, and urine and blood samples were obtained before contrast agent administration, with all but the history repeated afterward. The few exceptions to this procedure will be described. After contrast agent injection, patients were closely questioned regarding possible adverse reactions.

Patients with significant known or suspected liver disease, renal dysfunction, hemolytic anemia, uncontrolled seizures, pregnancy, and certain other medical conditions were excluded. The laboratory tests included a complete blood count, liver and kidney function tests, serum iron levels, and urinalysis. Substantial changes in laboratory results were defined as a change of more than $50 \%$ of the difference between the low and high values of the normal range. Vital signs were measured and a neurologic evaluation was done shortly before im- 
aging. Pre-contrast-enhancement images were obtained with both T1- and T2-weighted spin-echo sequences. Postcontrast images were obtained with T1-weighted spin-echo sequences in the same plane and with the same parameters as the precontrast study. Section thickness, section spacing, and the imaging plane(s) were chosen on the basis of clinical history in each case.

Gadodiamide injection was administered intravenously at a dose of approximately $0.1 \mathrm{mmol} / \mathrm{kg}$ (range, 08-0.11 mmol $/ \mathrm{kg}$ ). Immediately after contrast agent injection, approximately $5 \mathrm{~mL}$ of $0.9 \%$ normal saline was injected to ensure that all drug was cleared from the infusion tubing.

Vital signs were measured at approximately 5 , 30 , and 120 minutes after contrast agent injection. Two hours after injection, a repeat neurologic examination was performed. Approximately 24 hours after injection, repeat urine and blood samples were obtained. Discomfort or complaints of the patients were solicited and recorded throughout the study. Evaluation for adverse effects related to contrast agent administration was based on the parameters of Karch and Lasagna (2).

Images were reviewed by two radiologists (A.M.A., S.R.A.) experienced in interpretation of MR images. The efficacy of the agent was subjectively evaluated and a consensus reached. For purposes of this report, the following criteria were used to assess the efficacy of the contrast agent: (a) provision of additional information toward diagnosis, (b) increase in the confidence of the diagnosis, (c) detection of lesions not otherwise visible, and (d) provision of important information for greater characterization of lesions seen with other pulse sequences. In cases in which lesion enhancement was evident, the agent was judged to be of $(a)$ no help, (b) minor help, (c) moderate help, or (d) major help in diagnosis. In those cases that did not show abnormal enhancement, a judgment was made as to whether the agent was helpful in excluding suspected disease (eg, in cases of pituitary adenoma or acoustic neuroma).

Included in the study were 17 men and 13 women, ages ranging from 19 to 84 years (mean, 50 years). Images of the brain, pituitary gland, acoustic nerves, and/or foramen magnum were obtained in 20 patients; images of the spine were obtained in nine patients and of the skull base in one patient. The studies were requested for a variety of reasons, broadly categorized as follows (with some overlap): to rule out metastasis $(n=6)$, to rule out tumor in the cerebellopontine angle $(n=5)$, to rule out pituitary adenoma $(n=3)$, to rule out tumor $(n=5)$, to follow up tumor $(n=5)$, to differentiate disk abnormality from scar or tumor $(n=4)$, to rule out abscess $(n=1)$, and to follow up head and neck tumor $(n=1)$.

\section{- RESULTS}

\section{Study Protocol}

Eighteen patients were imaged on a 1.5-T system (Signa; GE Medical Systems, Milwaukee), 10 on a 0.35-T system (MT/S; Diasonics, Toshiba America MRI, South San Francisco, Calif), one on a 0.5-T system (HP; Picker, Cleveland), and one on both the 1.5-T and 0.35-T systems. Eleven patients received the contrast agent infusion over a 30-180-second duration (average, 101 seconds). The remainder received the injection in 30 seconds or less (average, 21 seconds).

All but one patient were imaged with $\mathrm{T} 1$ - and $\mathrm{T} 2$ weighted sequences before contrast agent injection and with $\mathrm{T} 1$-weighted sequences after injection; the exception was imaged with $\mathrm{T} 1$-weighted sequences before and after contrast agent injection. In one patient, the postcontrast neurologic examination was omitted, and in another patient the follow-up neurologic evaluation was performed after a delay of 24 hours.

The following laboratory tests were not performed before contrast agent injection: blood count in one patient, urinalysis in two patients, biochemistry studies in one patient, and serum iron level in one patient. In one additional patient, results of laboratory tests performed 2 months before the study were used and a serum iron level was not available; in another patient, the poststudy blood count was obtained after a 1 -week delay. Hence, in three patients serum iron levels were not obtained before contrast agent injection. Measurement of direct bilirubin was incomplete in the majority of patients because of difficulties related to transporting the blood specimens to the distant laboratory.

\section{Image Evaluation}

MR images after administration of gadodiamide injection (Figs $1-3$ ) showed enhancement patterns and imaging characteristics similar to what would be expected from the use of several existing gadolinium compounds (3-26) such as gadopentetate dimeglumine (Magnevist; Berlex Laboratories, Wayne, NJ) and gadolinium tetraazacyclododecanetetraacetic acid (DOTA), a macrocyclic gadolinium compound available in France, with contrast behavior similar to that of gadopentetate dimeglumine. In six subjects, contrast agent administration was of major diagnostic help, either revealing lesions not apparent without enhancement or providing important lesion characterization (Figs 1-3). In seven patients, this was true to a moderate degree, and in two subjects the contrast agent was thought to be of minor help. In 12 patients, the lack of abnormal enhancement patterns was important in excluding the presence of disease. In three patients, the agent was judged to be of no significant help, since the pattern of contrast enhancement (two patients) or the lack of enhancement (one patient) added no new information when compared with nonenhanced images.

\section{Clinical and Laboratory Evaluation}

Clinically, our patients experienced no major adverse reactions. No changes in vital signs were judged clinically significant. The average change in systolic blood pressure was $6 \mathrm{~mm} \mathrm{Hg}$, in diastolic blood pressure $5 \mathrm{~mm} \mathrm{Hg}$, in pulse rate four beats per minute, and in respiration two breaths per minute. Minor redness, swelling, or bruises at the injection site were noted in three patients; in two, this 

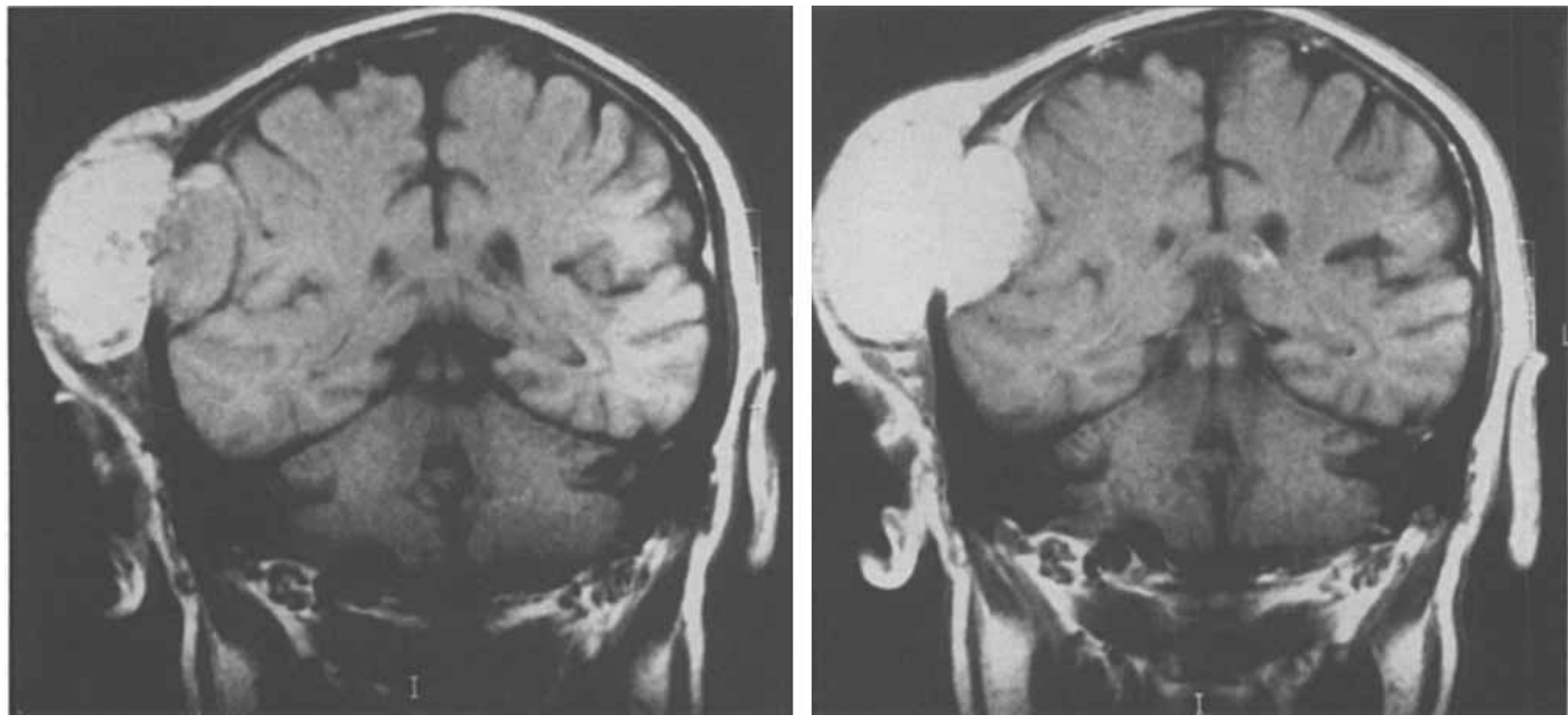

a.

b.

Figure 1. Thyroid carcinoma metastasis. T1-weighted images $(0.35 \mathrm{~T}$, TR msec/TE msec $=500 / 30)$ of a 70 -year-old man with a history of thyroid carcinoma. (a) Coronal precontrast image demonstrates a large right calvarial mass with high-signal-intensity areas suggestive of hemorrhage; the mass proved to be a metastasis from thyroid carcinoma. The major intracranial component is isointense with brain. (b) After contrast agent administration, the intracranial portion of the lesion is enhanced.

was probably related to extravasation of saline after contrast agent injection; in the third patient, the minor symptoms may have been related to infusion of the agent. Two patients complained of mild headaches; in both cases, these headaches resembled headaches they had experienced in the past, for which they were being evaluated. Three patients reported other minor symptoms thought to be of no practical consequence and likely not related to the physiologic effects of the contrast agent.

Nine patients had abnormal neurologic examination results, with findings usually related to the disease process for which they were referred for MR imaging. Except for the one patient who became drowsy as a result of two 10-mg doses of diazepam administered orally for claustrophobia, there were no changes in the neurologic examination results before and after contrast agent administration.

There were no substantial changes in laboratory results in 13 patients. One patient had a dramatic increase in platelet count (to 489,000 ), but the count was normal at retest several weeks later; the significance of the finding is unknown. Two patients had moderate increases in serum $\gamma$-glutamyltransferase to abnormal levels (the change was 34 IU/L for both; the pretest level in one of these patients was also abnormal). There were no consistent changes in the serum iron levels. In 12 patients the serum iron level increased, and in 15 it decreased; in three patients serum iron levels were not determined before contrast agent injection. In eight patients, the change was substantial. The average change in serum iron level was $5 \%$, and the average of the magnitude of changes in serum iron level was $32 \%$. The greatest change was $97 \%$, from 72 to $142 \mu \mathrm{g} / \mathrm{dL}(13 \mu \mathrm{mol} / \mathrm{L}$ to $25 \mu \mathrm{mol} / \mathrm{L}$ ) (both values are within normal limits). Six patients had abnormally high serum iron levels after contrast agent injection, and two had levels that were below normal. Of the six patients with elevated levels, two also had high serum iron levels before contrast agent administration.

\section{- DISCUSSION}

The sensitivity and specificity of MR imaging has improved with the introduction of gadolinium contrast agents (3-25). Enhancement of central nervous system lesions on $\mathrm{T} 1$-weighted images after gadolinium administration usually indicates an abnormal blood-brain barrier with viable blood supply or hypervascularity. Although MR images have proved highly sensitive for detection of lesions in, or closely related to, the central nervous system, experience with gadolinium contrast enhancement has shown that in some instances lesions that were not otherwise evident become apparent after contrast agent administration. For example, neuromas (14), pituitary microadenomas (16), and meningiomas $(13,15)$ are more readily seen after contrast agent administration. In addition, gadolinium-enhanced imaging has improved the delineation, and aided in the temporal characterization, of lesions with an actively disrupted blood-brain barrier. Such lesions include multiple sclerosis (21), abscess $(6,26)$, ischemia (22), and cerebritis (26). Finally, definition of the extent of tumors and their characterization can at times be improved, increasing diagnostic accuracy $(4-13,20)$. The absence of 


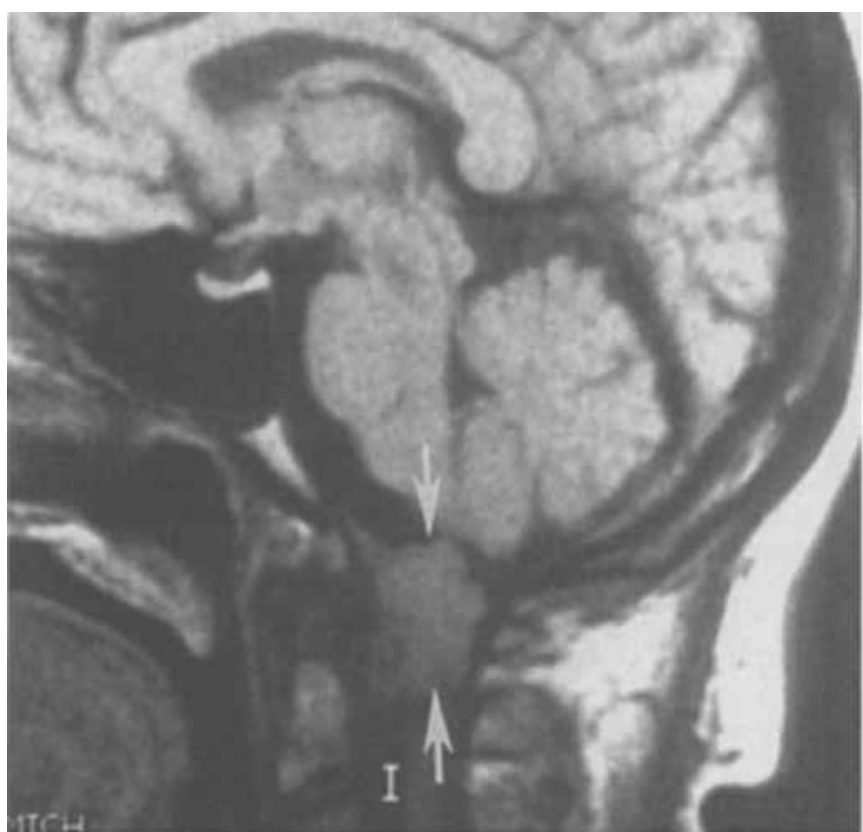

a.

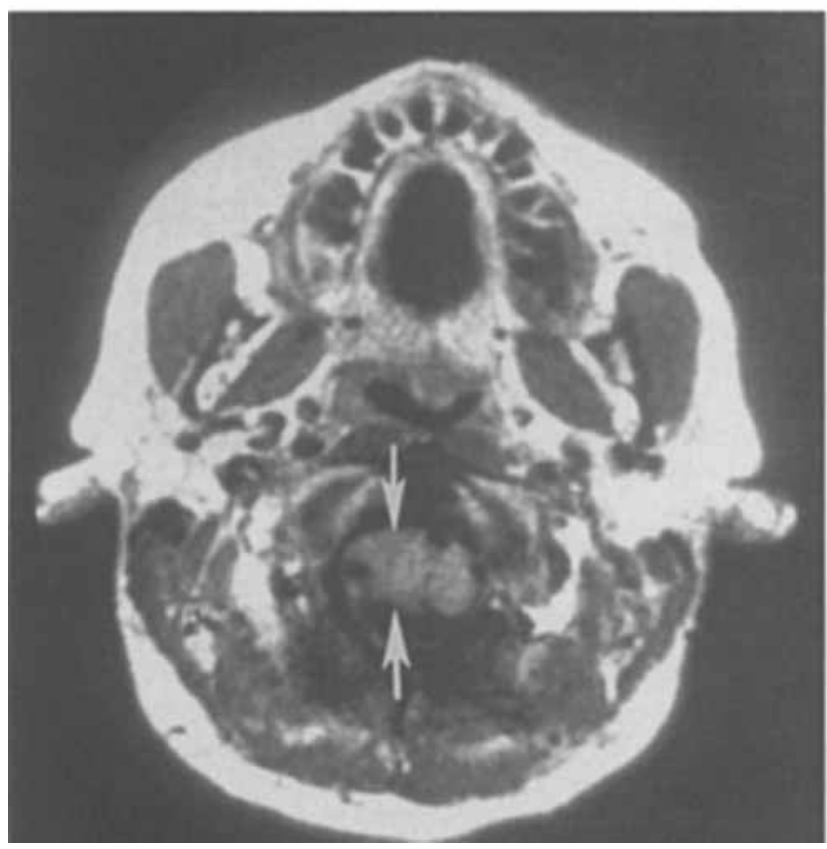

c.

Figure 2. Craniovertebral junction meningioma. T1-weighted images $(0.35 \mathrm{~T}, 500 / 40)$ of a 45 -year-old woman with neck pain demonstrate an enhancing lesion that proved to be a meningioma. (a) Precontrast sagittal image shows an extradural mass isointense with neural tissue (arrows), displacing the cervicomedullary junction. (b) Postcontrast image shows heterogeneous enhancement of the mass. (c) Precontrast axial image confirms the presence of the mass (arrows), which displaces the upper cervical spinal cord to the left. (d) Postcontrast Image again demonstrates enhancement of the mass.

areas of abnormal signal intensity on images obtained with all sequences (including $\mathrm{T} 1$-weighted sequences) after administration of a gadolinium compound provides valuable clinical information for excluding suspected disease. In cases in which questionable areas of signal-intensity abnormality

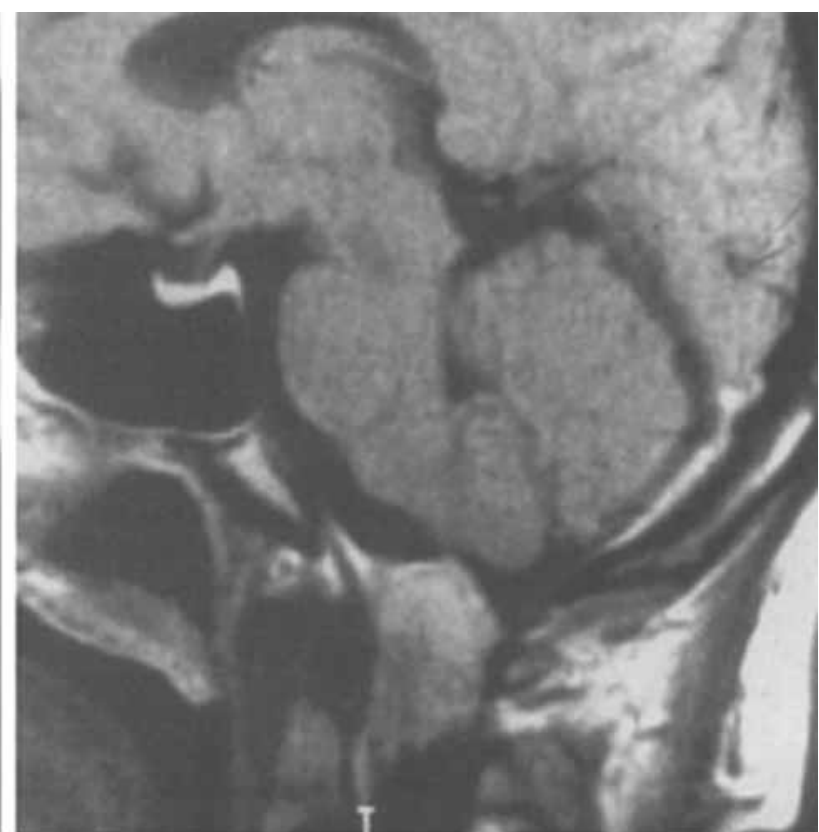

b.

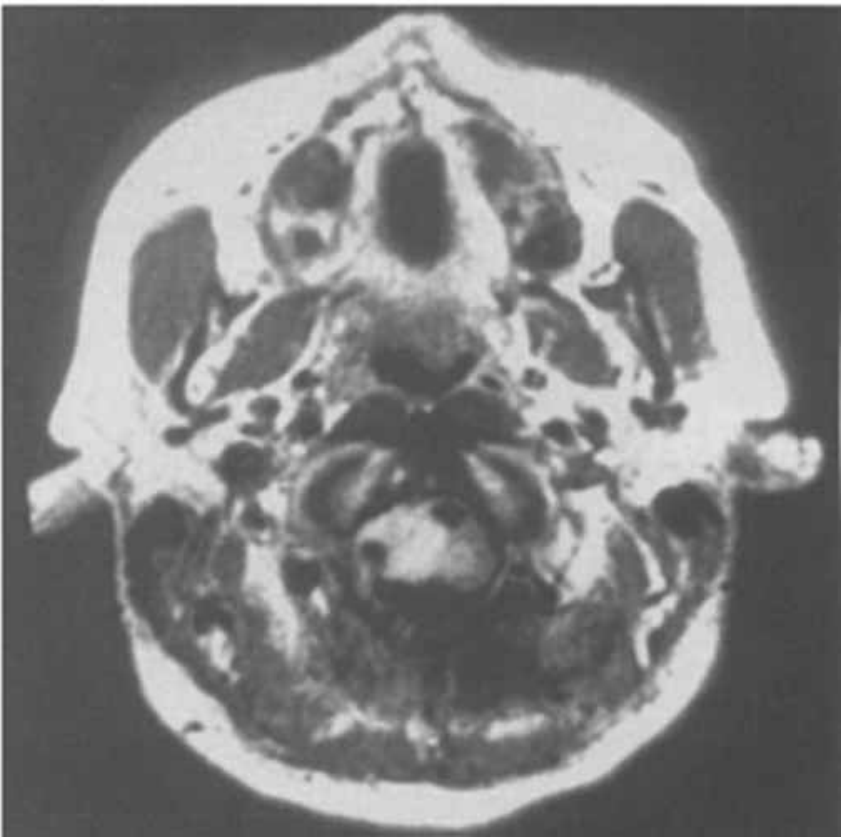

d.

are noted on previous unenhanced images, gadolinium can help determine the true nature of lesions or confirm their absence.

Gd-DTPA-BMA, like other gadolinium compounds, has unpaired electrons, resulting in paramagnetism that reduces the $\mathrm{T} 1$ and $\mathrm{T} 2$ of protons 


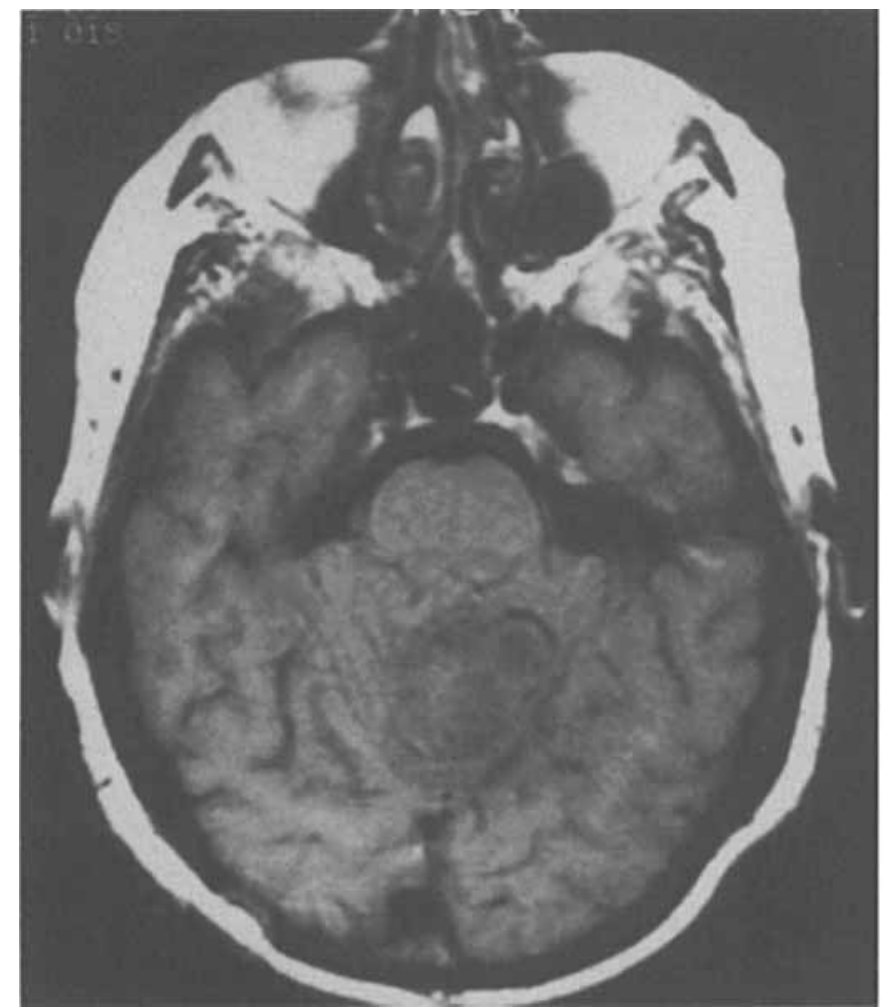

a.

Figure 3. Cystic astrocytoma. T1-weighted images $(0.35 \mathrm{~T}, 567 / 20)$ of a 68-year-old woman with a known cystic astrocytoma. (a) Precontrast axial image reveals a well-defined inhomogeneous mass in the superior vermis. (b) Postcontrast image demonstrates an irregular rim of enhancement with a central area of decreased signal intensity compatible with tumor surrounding a cystic or necrotic component.

$(27-29)$. Its relaxivity in water at $10 \mathrm{MHz}$ is 4.6 $\mathrm{mm}^{-1} \mathrm{sec}^{-1}$ for $\mathrm{T} 1$ and $5.1 \mathrm{~mm}^{-1} \mathrm{sec}^{-1}$ for T2 (1), which is similar to that of gadopentetate dimeglumine. Thus, at pharmacologically achieved concentrations, the agent can be expected to increase MR signal intensity on $\mathrm{T} 1$-weighted images at sites in the central nervous system where it accumulates because of lesion vascularity or breakdown of the blood-brain barrier. Animal and healthy human volunteer trials suggest that as a result of the nonionic structure (Salutar, data on file, 1989; 1), strong chelation, and reduced osmolarity of GdDTPA-BMA, it will be well tolerated in humans (1). In mice, the median lethal dose for gadodiamide injection is $34.4 \mathrm{mmol} / \mathrm{kg}$, which is substantially higher (ie, less toxic) than for gadolinium compounds currently avallable (Salutar, data on file, 1989; Berlex, Wayne, NJ, package insert for Magnevist, 1990).

Gadodiamide injection was found to be an efficacious agent, subjectively increasing diagnostic yield in the large majority of the patients studied. Its behavior was similar to what we have experienced and the literature has described regarding the available compounds gadopentetate dimeglumine and Gd-DOTA (3-26). We encountered no serious acute laboratory or clinical side effects in this limited number of patients that would preclude its use.

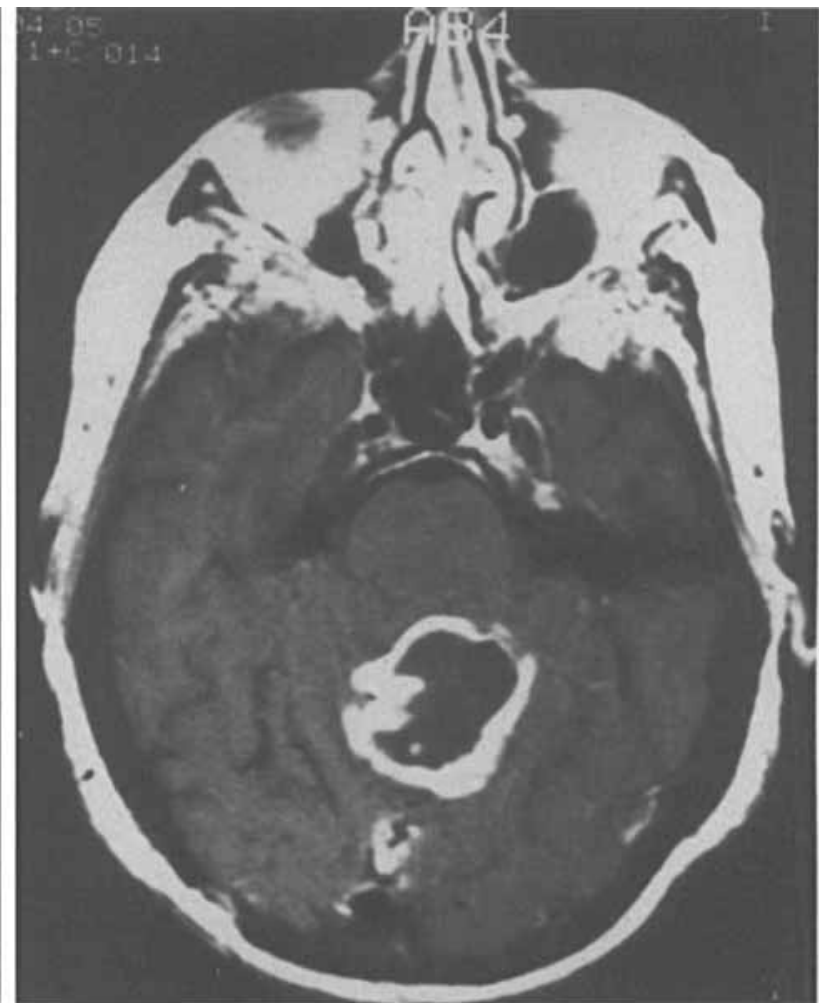

b. 
have resulted from use of contrast agent.

In conclusion, gadodiamide injection in this limited series proved to be a safe, well-tolerated, and efficacious MR imaging contrast agent for optimal evaluation of the central nervous system and adjacent structures, and the findings support its further development. The patterns of enhancement appear similar to those of the already approved agents gadopentetate dimeglumine and Gd-DOTA. If further experience and results from other centers corroborate these results, gadodiamide injection will likely prove to be a useful MR contrast agent, after regulatory approval.

Acknowledgments: The authors thank Steven guay, MD, PhD, Scott Rockledge, PhD, and Michelle Van Wagoner, BS. of Salutar for all their help; Jayshree Desai. MD, our research associate; and Douglas Quint, MD, for assistance with the manuscript.

\section{References}

1. O’Toole M. VanWagoner M. Broschart JL, Prasad R. Leese PT, Quay SC. Nonionic MRI contrast agents: a phase I clinical trial of the safety and tolerance of S-04 I injection (abstr). Magn Reson Imaging 1989; 7 (suppl 1): 174 .

2. Karch FE, Lasagna L. Adverse drug reactions: a critical review. JAMA 1975; 234:1236-1241.

3. Laniado M, Weinmann HJ, Schorner W, Felix R, Speck U. First use of Gd DTPA/dimeglumine in man. Physiol Chem Phys Med NMR 1984; 16:157-165.

4. Carr DH, Brown J, Bydder GM, et al. Gadolinium-DTPA as a contrast agent in MRI: initial clinical experience in 20 patients. AJR 1984; 143:215-224.

5. Runge VM, Schörner W, Niendorf HP, et al. Initial clinical evaluation of gadolinium DTPA for contrast-enhanced magnetic resonance imaging. Magn Reson Imaging $1985: 3: 27-35$.

6. Bydder GM. Clinical application of gadolinium-DTPA. In: Stark DD, Bradley WG, eds. Magnetic resonance imaging. St. Louis: Mosby, 1988; 182-200.

7. Stack JP, Antoun NM, Jenkins JPR, Metcalfe R, Isherwood I. Gadolinium-DTPA as a contrast agent in magnetic resonance imaging of the brain. Neuroradiology 1988: 30:145-154.

8. Bauer WM, Fenzl G, Vog! TH, Fink U, Lissner J. Indications for the use of Gd-DTPA in MRI of the central nervous system: experiences in patients with cerebral and spinal diseases. Invest Radiol 1988: 23(suppl 1):52865288

9. Felix R, Schörner W, Laniado M, et al. Brain tumors: MR imaging with gadolinium-DTPA. Radiology 1985 : 156:681-688.

10. Claussen C, Laniado M, Schörner W, et al. GadoliniumDTPA in MR imaging of glioblastomas and intracranial metastases. AJNR 1985; 6:669-674.

11. Brant-Zawadzki M, Berry 1, Osaki L. Brasch RC, Murovic J, Norman D. Gd-DTPA in clinical MR imaging of the brain. I. Intraaxial lesions. AJR 1986; 147:1223-1230.

12. Berry I, Brant-Zawadzki M, Osaki L, Brasch R, Murovic J, Newton TH. Gd-DTPA in clinical MR of the brain. II Extraaxial lesions and normal structures. AJR 1986 $147: 1231-1235$.

13. Bydder GM, Kingsley DPE, Brown J, Niendorf HP, Young IR. MR imaging of meningiomas including studies with and without gadolinium-DTPA. J Comput Assist Tomogr 1985: 9:690-697.

14. Curati WL. Graif M, Kingsley DPE, Niendorf HP, Young IR. Acoustic neuromas: Gd-DTPA enhancement in MR imaging. Radiology 1986; 158:447-451.

15. Breger RK, Papke RA. Pojunas KW, et al. Benign extraaxial tumors: contrast enhancement with Gd-DTPA. Radiology 1987: 163:427-429.

16. Dwyer AJ, Frank JA, Doppman JL, et al. Pituitary ade nomas in patients with Cushing disease: initial experience with Gd-DTPA-enhanced MR imaging. Radiology 1987: 163:421-426.

17. Russell EJ, Gesemis GK. Johnson CE, et al. Multiple cerebral metastases: detectability with Gd-DTPA-enhanced MR imaging. Radiology 1987; 165:609-617.

18. Powers TA, Partain CL, Kessler RM, et al. Central nervous system lesions in pediatric patients: Gd-DTPA-enhanced MR imaging. Radiology 1988: 169:723-726.

19. Bird CR, Draver BF, Medina M, Rekate HL, Flom RA. Hodak JA. Gd-DTPA-enhanced MR imaging in pediatric patients after brain tumor resection. Radiology 1988; 169:123-126.

20. Bydder GM, Brown J, Niendorf HP, Young IR. Enhancement of cervical intraspinal tumors in MR imaging with intravenous gadolinium-DTPA. J Comput Assist Tomogr 1985; 9:847-851

21. Gonzalez-Scarano F, Grossman RJ, Galetts S, Atlas SW, Silberberg DH. Multiple sclerosis disease activity correlates with gadolinium-enhanced magnetic resonance imaging. Ann Neurol 1987: 21:300-306.

22. Virapongse C, Mancuso A. Quisling R. Human brain infarcts: Gd-DTPA-enhanced MR imaging. Radiology 1986; $161: 785-794$

23. Bousquet JC. Saini S. Stark DD. et al. Gd-DOTA: characterization of a new paramagnetic complex. Radiology $1988 ; 166: 693-698$

24. Magerstadt M, Gansow OA, Brechbiel MW, et al. Gd(DOTA): an alternative to Gd(DTPA) as a $T 1,2$ relaxation agent for NMR imaging or spectroscopy. Magn Reson Med 1986; 3:808-812.

25. Parizel PM, Degryse HR, Gheuens J, et al, GadoliniumDOTA enhanced MR imaging of intracranial lesions. $J$ Comput Assist Tomogr 1989; 13:378-385.

26. Grossman RI. Wolf G. Biery D, et al. Gadolinium-enhanced nuclear magnetic resonance images of experimental brain abscess. J Comput Assist Tomogr 1984; 8:204-207.

27. Lauffer RB, Brady TJ. Preparation and water relaxation properties of proteins labeled with paramagnetic metal chelates. Magn Reson Imaging 1985; 3:1 1-16.

28. Goldstein EJ, Burnett KR, Hansell JR, et al. Gadolinium DTPA (an NMR proton imaging contrast agent) chemical structure, paramagnetic properties and pharmacokinetics. Physiol Chem Phys Med NMR 1984: 16:97-104.

29. Koenig SH, Spiller M, Brown RD III, Wolf GL. Relaxation of water protons in the intra- and extracellular regions of blood containing Gd(DTPA). Magn Reson Med 1986; 3:791-795.

30. Bydder GM. Clinical application of gadolinium-DTPA. In: Stark DD. Bradley WG, eds. Magnetic resonance imaging. St. Louis: Mosby, 1988; 199

31. Niendorf HP, Seifert $W$. Serum iron and serum bilirubin after administration of Gd-DTPA-dimeglumine: a pharmacologic study in healthy volunteers. Invest Radiol 1988; 23 (suppl 1):S275-S280. 